\title{
A Quadcopter Controller to Maintain Radio Link Quality
}

\author{
Ayyoob Hamza \\ University of Colombo \\ Sri Lanka \\ ayyoob@scorelab.org \\ Venkat lyer \\ Uppsala University \\ Sweden \\ venkatraman.iyer@it.uu.se
}

\author{
Chamath Keppitiyagama \\ University of Colombo \\ Sri Lanka \\ chamath@ucsc.lk \\ Kasun Hewage \\ Uppsala University \\ Sweden \\ kasun.hewage@it.uu.se
}

\author{
Kasun De Zoysa \\ University of Colombo \\ Sri Lanka \\ kasun@ucsc.lk \\ Thiemo Voigt \\ Uppsala University \\ SICS Swedish ICT \\ thiemo.voigt@it.uu.se
}

\begin{abstract}
The excellent maneuverability and the availability of a large number of sensors including good quality video cameras make quadcopters attractive for surveillance systems. Most video surveillance systems need a real-time high quality video stream from the cameras on the quadcopter to the base station which requires a stable and reliable radio link. Moreover, to ensure a controlled flight, it is essential to maintain a good quality radio link from the base station to the quadcopter. The quality of this link depends on the distance between base station and quadcopter as well as the ambient noise. Towards this end, we present a controller to hover a quadcopter at the maximum distance from the base station while maintaining the link quality at a given value indicated by the Signal to Noise Ratio (SNR). There are existing solutions in the field of robotics that incorporate the concept of link aware mobility. However, to the best of our knowledge, this is the first link-quality aware mobility implementation on a quadcopter.
\end{abstract}

\section{Keywords}

Quadcopter Controller, Autonomous, Link Aware Mobility, Signal to Noise Ratio

\section{INTRODUCTION}

Surveillance has entered a new era with the introduction of unmanned aerial vehicle (UAVs) such as quadcopters that combine sensing, flying, and communication. Most UAVs can capture high resolution images and transmit them to a base station in real-time. This requires a good radio link between the UAV and the base station.

There are surveillance systems that require sending UAVs from a control station (base station) as close as possible to the location under surveillance. Sending quadcopters deep into a jungle to get video streams for wildlife monitoring is an example of such an application. The maximum distance

Permission to make digital or hard copies of part or all of this work for personal or classroom use is granted without fee provided that copies are not made or distributed for profit or commercial advantage and that copies bear this notice and the full citation on the first page. Copyrights for third-party components of this work must be honored. For all other uses, contact the Owner/Author.Copyright is held by the owner/author(s). DroNet'15, May 19 2015, Florence, Italy.

ACM 978-1-4503-3501-0/15/05.

http://dx.doi.org/10.1145/2750675.2750678. that a quadcopter can travel from the base station depends on the minimum Signal to Noise Ratio (SNR) requirement of the application since the signal strength decreases with the distance. To support similar applications, we consider the problem of hovering a quadcopter at the maximum possible distance from a base station while still maintaining a given SNR.

Several factors such as the transmission power, antenna type, antenna gain, environmental noise, and interference affect the quality of the radio link between a base station and a quadcopter. However, most quadcopter applications do not consider these factors and they tend to use an empirical preset measurement for the maximum distance. Such a preset maximum distance is not suitable for the surveillance applications discussed above.

In this paper, we present a controller that hovers a quadcopter at the maximum possible distance from a base station while maintaining a given minimum SNR. Our controller learns how the SNR varies with the distance while flying and adapts to achieve the target SNR. We present the evolution of the controller from an initial design based on the results of several experiments and we also evaluate the controller's ability to achieve its target.

\section{RELATED WORK}

Link quality aware mobility is a concept that has been used in the field of robotics. Dixon et al. [6] and Khim Yee [8] have proposed solutions to achieve link aware mobility. Both approaches use SNR as the signal metric and an Extremum Seeking (ES) based real-time adaptive controller to maintain the link. The ES model is similar to a bicycle-like kinematics that can only be used on vehicles that can either move forward or backward. An extremum seeking controller is appropriate if we want the quadcopter to move only in one direction. However, due to wind and other external influences the quadcopter could deviate from its current heading.

Freitas et al. [9], Rohde et al. [10], Daniel et al. [5] and Goddemeier et al.'s [7] approaches have used Received Signal Strength Indicator (RSSI) as a signal quality metric for maintaining link on UAVs. Signal strength alone does not indicate the quality of a wireless link. The link quality also depends on the noise in the environment and the radio interference. Therefore SNR is a better metric to measure the link quality [3]. 
Though link quality aware mobility concepts have already been used in robotics, to the best of our knowledge, ours is the first SNR based link aware mobility implementation for quadcopters.

\section{THE CONTROLLER}

The goal of the controller is to autonomously guide the quadcopter to a position with the target SNR and keep it hovering there. The controller dynamically adapts to the changes in the environment and it has the ability to use the prior knowledge about the SNR variations in the environment. We use the principles of cognitive based controllers [4] in designing our controller to achieve these goals. Such a controller estimates the state based on the relevant data, performs some actions on the plant, which are based on the past information about the environment and also learns and adapts to the changes in the environment.

Our controller has four main components and the interactions between these components are depicted in Figure 1.

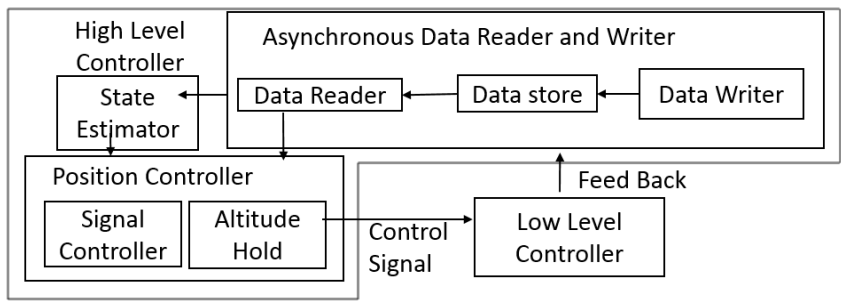

Figure 1: System architecture of the controller

Low Level Controller: The low level controller acts as an open loop control system which is a mere representation of the quadcopter. The low-level controller derives its input from the high level controller. This input consists of forward, lateral, vertical and angular speeds that collectively change the state of the quadcopter. The velocity, orientation and GPS information of the quadcopter is sent back to the high level controller as navigation data.

Asynchronous Data Reader and Writer: The data writer extracts yaw (rotation in vertical axis), altitude, velocities and GPS data from the low level controller and writes this data into a data store. The data reader reads the data from the data store and triggers the state estimator to predict the state of the quadcopter.

State Estimator: We use the state estimator to identify the position of the quadcopter from the base station. We use an Extended Kalman Filter (EKF) that combines GPS and dead reckoning to find the position of the quadcopter [11]. The GPS data is often delayed and the observations are noisy. In dead reckoning, the error increases with the distance. EKF uses both the GPS and dead reckoning to give a better estimate of the position. We use dead reckoning as a prediction step and the GPS location as a correction step of the EKF. However, a GPS calculates its location on the world coordinate system and dead reckoning predicts the location on the local coordinate system.

The quadcopter moves in a 3D space. However, in this work it either moves away or towards the base station in a straight line at a fixed altitude in an obstacle-free environment. Note that our controller does not handle obstacles. To simplify the controller design, we use a $3 \mathrm{D}$ coordinate system relative to the base station and the initial placement of the quadcopter as the origin of this coordinate system. We denote this as the base coordinate system. By proposing this coordinate system we have reduced the problem of finding the maximum point on the $\mathrm{X}$ axis of base coordinate system $(X, Y=0, Z=$ altitude, $Y a w=0)$ for a given SNR value. Figure 2 depicts the process of predicting the state on the base coordinate system. The prediction step of the EKF

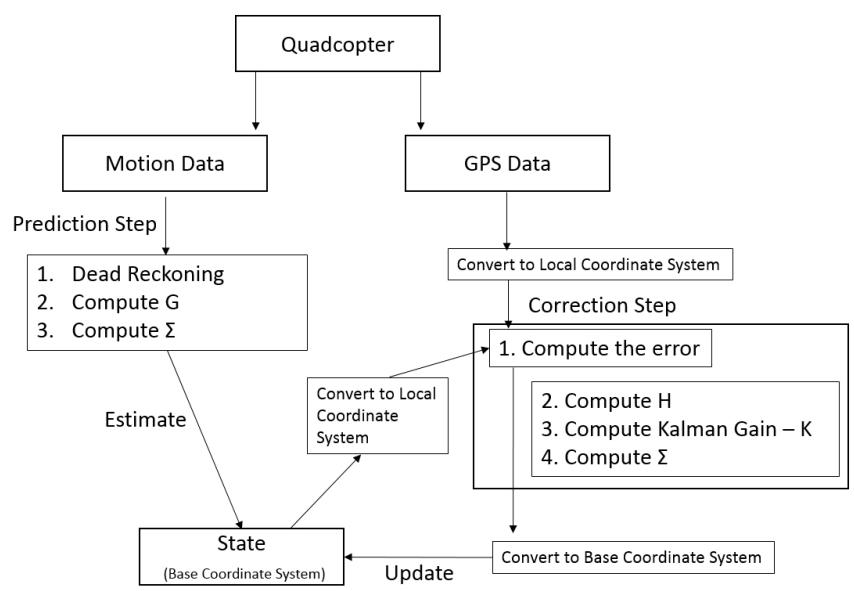

Figure 2: Computational flow of state estimation using EKF

runs whenever the controller receives odometry data. This data is on local coordinates and must be transformed into the base coordinate system. While computing the current state, the prediction step also computes the EKF parameters: the transition matrices $(G)$ and the covariance estimate $(\Sigma)$.

Prediction Step: $\mathrm{g}\left(\mu_{t-1}, u_{t}\right)$ is a motion function that depends on previous position $\left(\mu_{t-1}\right)$ and control signal $u_{t}$. We are considering the motion in a $2 \mathrm{D}$ space with a given altitude. Therefore, the Motion Function (x, y , yaw $(\psi)$ ) is:

$$
\begin{gathered}
\bar{\mu}_{t}=g\left(p, u_{t}\right)=\left(\begin{array}{c}
x+(\cos (\psi) \dot{x}-\sin (\psi) \dot{y}) \Delta t \\
y+(\sin (\psi) \dot{x}+\cos (\psi) \dot{y}) \Delta t \\
\psi+\dot{\psi} \Delta t
\end{array}\right) \\
\bar{\Sigma}_{t}=G_{t} \Sigma G_{t}^{T}+Q \text { with } G_{t}=\left.\frac{\delta g\left(p, u_{t}\right)}{\delta p}\right|_{p=\mu_{t-1}}
\end{gathered}
$$

$Q$ is the motion noise covariance.

The correction step runs whenever GPS data is received. The correction step computes the error by using the current state and the measured state (GPS). The current state is, however, on the base coordinate system and GPS is on the world coordinate system (relative to the base station but y axis along the true north). Therefore, we use both measurements to transform them into the local coordinate system of the quadcopter. The error $(e)$, observation matrices $(H)$, Kalman gain $(K)$ and $(\Sigma)$ are computed to update the error on the current state.

Correction Step:

$$
\begin{gathered}
\mu_{t}=\bar{\mu}_{t}+K_{t}(e) \\
e=z_{t}-h\left(\bar{\mu}_{t}\right)
\end{gathered}
$$


Equation 4 illustrates the calculation of measurement error, in which $z_{t}$ denotes the position calculated using GPS [2] and $\mathrm{h}\left(\bar{\mu}_{t}\right)$ denotes the observation function that is used to calculate the perceived position. Kalman gain can be computed using Equation 5.

$$
K_{t}=\bar{\Sigma}_{t} H_{t}^{T}\left(H_{t} \bar{\Sigma}_{t} H_{t}^{T}+R\right)^{-1} \text { with } H_{t}=\left.\frac{\delta h\left(p, u_{t}\right)}{\delta p}\right|_{p=\bar{\mu}_{t}}
$$

Position Controller: The position controller is the core component of the controller. The signal controller drives the quadcopter to the desired link quality. The altitude hold makes certain that the quadcopter flies in a given altitude.

Altitude Hold: The altitude hold is use to maintain the quadcopter at a given altitude. We use a Proportional Derivative (PD) controller to achieve this objective. The vertical speed is then calculated using the altitude error and a vertical speed transfer function. This speed is sent to the low level controller as a control signal to drive the quadcopter.

Signal Controller: Figure 3 illustrates the basic design

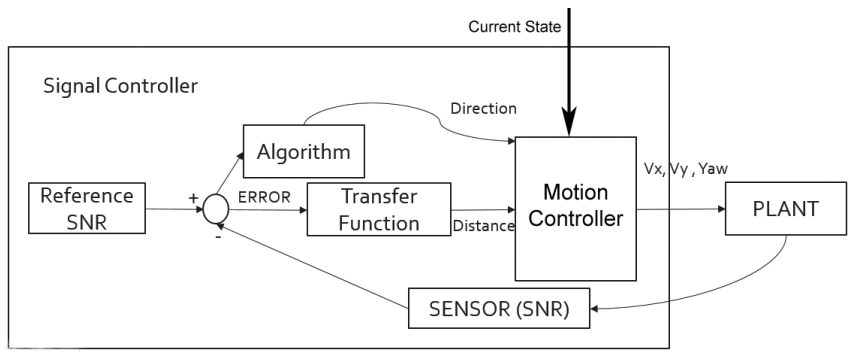

Figure 3: Design for the Signal Controller

of the signal controller. The plant only understands the signal in velocity. We require a suitable model to map the SNR to the velocity to achieve the target SNR. Distance is the integration of the velocity. Therefore, we propose a transfer function and an algorithm to decide the distance and the direction the quadcopter has to move to achieve the target SNR. We use a motion controller to move the quadcopter to achieve the target position when the target distance and direction is calculated on the base coordinate system.

Transfer Function: We use a learning mechanism to map the SNR to the distance. Algorithm 1 illustrates the steps

\footnotetext{
Algorithm 1 Modeling Transfer function

Step 1: Collect SNR vs Distance data on a fixed altitude using the quadcopter

Step 2: Regression over the data

$$
\text { Distance } \propto\left(\frac{1}{S N R}\right)^{\alpha}
$$

Step 3: Create SNR to Distance Map (SNRDM)

of the transfer function. We use a fixed distance based controller to collect the data in a test run. For the purpose of regression, we consider the model given in Equation 6 . Note that this model is only used for the purpose of initial estimation and the outcome of this model is applicable for that tested environment. Once the coefficients are found, a $\mathrm{SNR}$ to distance map (SNRDM) is created for the range of
}

minimum SNR to maximum SNR. A linear approximation is applied on the distances from the current SNR to the target SNR on SNRDM to compute the distance to be moved to achieve the target SNR.

Direction Algorithm: We use a simple algorithm to calculate the direction of the flight (Algorithm 2). Since the calculations are done with reference to the $X$ axis on base coordinate system, the quadcopter direction has only three states $(+,-, 0)$.

\begin{tabular}{l}
\hline Algorithm 2 Algorithm to find the flight direction \\
IF SNR < Target SNR : MoveTowardsTheBaseStation(-) \\
Else IF SNR > Target SNR: MoveAwayFromBaseStation(+) \\
Else : Hover $(0) ;$
\end{tabular}

Motion Controller: Once the distance (d) and direction $(\lambda)$ is found then we calculate the target $x, y$ and yaw. This is illustrated in Figure 4.

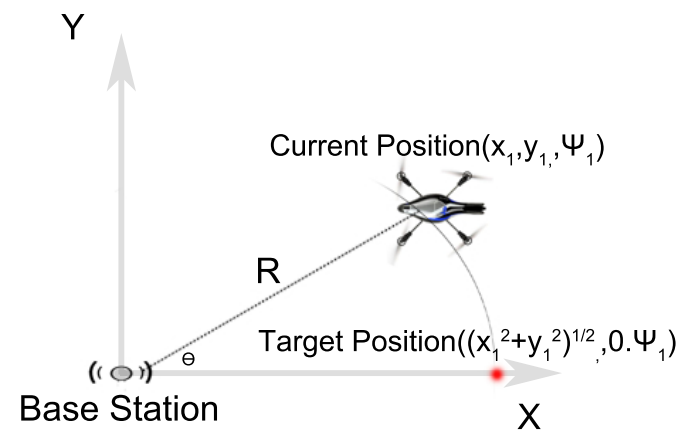

Figure 4: Correction to the drift

$$
\begin{gathered}
\text { CurrentState }=\left(x_{t}, y_{t}, \psi_{t}\right)^{T} \\
\text { TargetState }=\left(\left(\left(\left(x_{t}\right)^{2}+\left(y_{t}\right)^{2}\right)^{1 / 2}\right)+(\lambda * d), 0, \psi_{s}\right)^{T} \\
{\left[\begin{array}{lll}
x & y & \text { yaw }
\end{array}\right]^{T}=\text { TargetState }- \text { CurrentState }}
\end{gathered}
$$

$\psi_{s}$ is the target orientation.

Equation 8 calculates the target coordinates for the desired SNR. Though the quadcopter should move along the $X$ axis, it could drift away due to wind. However, the SNR of the current position can be achieved in the same distance along the $X$ axis. This assumption is true for the same radius with small $\theta$. Using this assumption we can find the distance to move from the current position on 3 axes by using the Equation 9. We can transform these distances into the local coordinate system. These local distances of $x, y$ and yaw values are fed to their own PD controller for each axis.

\section{REFINING THE CONTROLLER}

We implemented a prototype controller for the AR-Drone 2.0 [1]. In this prototype we run the controller on a laptop and we send control signals and receive navigation data from the quadcopter over a WiFi link. Our intention is to run the controller on the quadcopter itself. However, to ease the debugging and testing we run the controller in a laptop in this prototype.

We conducted several experiments to evaluate the effectiveness of the controller and to refine the design. 


\subsection{The Yaw Controller}

So far we have assumed that the SNR at a given position is independent of the orientation of the quadcopter since it uses an omni directional antenna. If this assumption is true, then there is no need for a yaw controller. We conducted an experiment to ascertain the validity of this assumption.

We placed the quadcopter on a rotatable disk to conduct this experiment. We rotated the disk in steps of approximately 5-25 degree angles for a complete circle and we recorded the SNR at each step. We used 30 such iterations for this experiment. In a single full rotation, we introduced an interval of approximately 1 second between successive steps to ensure that each reading reaches a stable point.

We recorded a mean SNR of 33.71 with a standard deviation of 2.099 with the rotation. However, the mean SNR without rotation was 36.49 with a standard deviation of 0.501 . We used a two sample t-test with $\mathrm{z}$ approximation to evaluate the effect of rotation on the SNR. The results indicate that there is a significant impact of the orientation of the quadcopter on the SNR. Therefore to mitigate this effect on SNR, we decided to use a yaw controller to maintain the quadcopter at a fixed orientation throughout the flight.

\subsection{Knowledge Module and Learning Module}

The knowledge module provides prior information on how the SNR changes with the distance to the transfer function on the signal controller. This prior knowledge is used to give a hint to the controller on the distance to travel to achieve the target SNR. We perform experiments to investigate how best to model this prior knowledge. Towards this end, we set a target distance and recorded the distance traveled from the origin and the SNR against the time. We repeated this for 10 flights.

Figure 5 depicts how the distance and SNR varies with time for a flight. It is evident that the SNR readings have an unusual behavior after passing the target distance for the first time. The motion controller applies a sequence of minor corrections at values close to the target and this results in several sudden changes in the velocity. The orientation of the quadcopter and its antenna changes as a reaction to these changes in the velocity. This is the reason for the fluctuations in the SNR near the target.

We cannot completely eliminate spurious SNR values since the controller applies continuous error corrections (and hence sudden velocity changes) as reactions to the drift and the altitude changes. Due to this there are some spurious values in the SNR readings. To avoid this we used the medians to reduce the effect of the outliers on the distance corresponding to a particular SNR.

We assume that the maximum distance that can be traveled while still maintaining a given SNR can be modeled by the Equation 6, which is related to the free space path loss model. We conducted a univariate regression on the data set for different values of $\alpha$ in the Equation 6 and we present the results in the Table 1.

The R-Squared goodness of fit statistical measure for the regression line when $\alpha=1$ is $78.6 \%$. Yet, the R-Squared does not indicate that the model has a good fit. Therefore we further looked into the residual behavior on independence and normalize. We found that there was bias in the model. We conducted further analysis to test whether different $\alpha$ values improves the goodness of the fit.

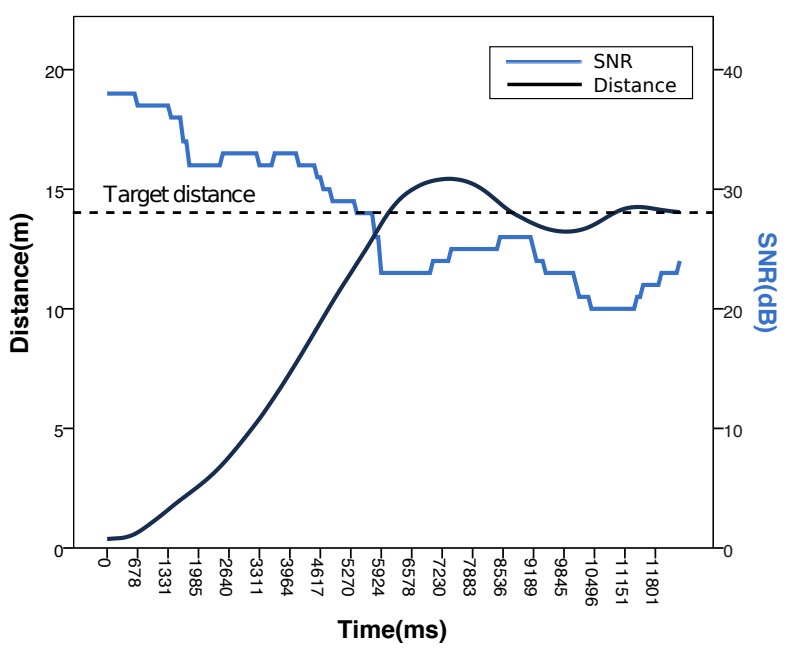

Figure 5: SNR and distance variation over time

\begin{tabular}{|c|c|c|c|}
\hline$\alpha$ & R squared & $\begin{array}{c}\text { Highest Degree } \\
\text { Coefficient (1/SNR) }\end{array}$ & Intercept \\
\hline 0.33 & $78.7 \%$ & 233.323 & -67.134 \\
\hline 0.5 & $78.7 \%$ & 278.222 & -42.023 \\
\hline 1 & $78.6 \%$ & 788.054 & -17.618 \\
\hline 2 & $77.4 \%$ & 12340.402 & -5.344 \\
\hline 3 & $75 \%$ & 249524.745 & -1.190 \\
\hline
\end{tabular}

Table 1: Goodness of Fit for different $\alpha$

When $\alpha$ increases the value for R-Squared decreases. By looking at the coefficient it is also evident that with the increase of $\alpha$ the coefficient has increased and the weight for $1 / \mathrm{SNR}$ has decreased. An increase of $\alpha$ makes the model more complex compared to the improvement it does. When decreasing the $\alpha$ it does not make much difference on residuals. Therefore, for simplicity, we set $\alpha$ to 1 , as setting it to higher values does not bring much improvement in observed residuals.

Link quality changes over time [3]. Therefore, different trials may have different characterization of SNR over distance. We use the regression on the data for each specific flight individually we get a better goodness of fit (Table 2).

\begin{tabular}{|c|c|}
\hline R squared & $88.8 \%$ \\
\hline Adjusted R squared & $87 \%$ \\
\hline \hline Parameter & Significance F-Test \\
\hline Intercept & 0.000 \\
\hline Coff. 1/SNR & 0.000 \\
\hline
\end{tabular}

Table 2: Results for the regression model with $\alpha=1$

This analysis shows that one model does not fit all flights. However, a model based on the historical data can be used to provide an initial estimation or a hint. The results also suggest that the maximum distance corresponding to a given SNR varies from flight to flight and we cannot always achieve the maximum distance with a fixed-distance based approach. Therefore, for each flight an individual SNR to distance model, a learning model, is needed. 
The intent of the learning module is to improve on the prediction of the target distance ( $\mathrm{x}$ coordinate of base coordinate system) for the reference SNR, using the data gathered during the flight. To achieve this we have to solve the problem of the spurious SNR values mentioned before. The median filter we use to address this problem is not suitable for an on-line learning algorithm due to its space complexity. Therefore, we use the harmonic mean.

The learning model is still vulnerable to sudden change of orientation. To reduce this effect we use a filter, and accept the currently observed SNR, denoted as $S N R_{t}$, only if the following condition holds:

$$
S N R_{t-1}+C S N R>S N R_{t}
$$

Here, CSNR is the ceiling on the standard deviation of the SNR. CSNR is the maximum SNR change that can occur from the current SNR reading. This minimizes the sudden fluctuations in the SNR readings.

\section{EVALUATION}

In this section we analyze the performance of the controller. For this evaluation we collected data from 22 flights, on different days and times. We fly the quadcopter at a fixed altitude of 7 meters. We use the target SNRs of $25 \mathrm{~dB}$ and $30 \mathrm{~dB}$. Table 3 shows several descriptive statistics of these flights. The time shown in the table is the time to reach the target SNR. After the quadcopter has reached the target SNR we kept it flying for another three minutes.

\begin{tabular}{|c|c|c|c|c|c|}
\hline $\begin{array}{l}\text { Target } \\
\text { SNR } \\
d(d B)\end{array}$ & $\begin{array}{c}\text { Prior } \\
\text { Knowl- } \\
\text { edge }\end{array}$ & Parameter & $\begin{array}{c}\begin{array}{c}\text { No. } \\
\text { of } \\
\text { Flights }\end{array} \\
\end{array}$ & Mean & $\begin{array}{l}\text { Std. } \\
\text { Dev }\end{array}$ \\
\hline \multirow{4}{*}{25} & \multirow{2}{*}{ No } & Time (s) & \multirow{2}{*}{5} & 78.899 & 26.086 \\
\hline & & Distance(m) & & $18.55^{r}$ & 4.724 \\
\hline & \multirow{2}{*}{ Yes } & Time (s) & \multirow{2}{*}{7} & 40.718 & 13.816 \\
\hline & & Distance $(\mathrm{m})$ & & 18.731 & 6.364 \\
\hline \multirow{4}{*}{30} & \multirow{2}{*}{ No } & Time(s) & \multirow{2}{*}{5} & 35.212 & 32.252 \\
\hline & & Distance $(\mathrm{m})$ & & 5.712 & 1.912 \\
\hline & \multirow{2}{*}{ Yes } & Time $(\mathrm{s})$ & \multirow{2}{*}{5} & 27.598 & 20.365 \\
\hline & & Distance $(\mathrm{m})$ & & 6.278 & 1.699 \\
\hline
\end{tabular}

Table 3: Time-Distance Variation for given SNR values (with \& without prior knowledge)

\section{Evaluation of the Controller:}

The controller drives the quadcopter along the $\mathrm{X}$ axis of the base coordinate system while maintaining $\mathrm{Y}=0 \mathrm{~m}$, altitude $\mathrm{Z}=7 \mathrm{~m}$, and the $\mathrm{Yaw}=0$ degrees.

Table 4 shows the movements along the $\mathrm{Y}, \mathrm{Z}$ axes and the changes in the orientation of a flight. It shows that the $\mathrm{Y}$ axis mean is very close to zero and the standard deviation is also small $(45 \mathrm{~cm})$. The $\mathrm{Z}$ axis is also close to the target value of 7 meters and the standard deviation is $12 \mathrm{~cm}$. These values show that the controller is effective in maintaining the trajectory of the quadcopter. However, the yaw controller is not as effective. The yaw motion has a mean of -0.11 radian (-6.68 degree) and a standard deviation of 0.493 (28.26 degree). This shows that the yaw controller struggles to maintain the orientation.

The Yaw motion is relatively very slow compared to other movements. Therefore, it takes a long time to regain its

\begin{tabular}{|c|c|c|c|c|}
\hline & Min & Max & Mean & Std. Dev \\
\hline Y Axis(m) & -2.605 & 1.532 & -0.054 & 0.452 \\
\hline Z Axis(m) & 6.460 & 7.427 & 7.002 & 0.122 \\
\hline Yaw(rad) & -3.992 & 21.294 & -0.116 & 0.493 \\
\hline
\end{tabular}

Table 4: Ability to maintain the trajectory the orientation.

orientation. A change in Yaw affects the SNR readings. Therefore, we use a CSNR smoothing filter.

We conducted further analysis to understand the behavior of the controller on the $\mathrm{X}$ axis. We observed that all of the 22 flights achieved the target SNR. However, once it reaches the target, the quadcopter moves back and forth from this point indicating the fluctuations in the SNR readings. We calculated the mean SNR and the standard deviation once the quadcopter has reached the target to estimate its capability to be within the target SNR. However these metrics do not show whether the quadcopter is hovering as expected. Therefore, we introduce the metric Hover Measure $(\gamma)$ that describes the controller's effectiveness in keeping the quadcopter hovering at the target point rather than moving back and forth.

$$
\text { Hover Measure }(\gamma)=\frac{\text { Total hovering time }}{\text { Total flying time }} \times 100 \%
$$

Note that Equation 10 is only applicable after the quadcopter has reached the target SNR for the first time. In this equation Total flying time indicates the total time it flew after reaching the target SNR and the Total hovering time is the sum of all time-periods for which the drone was hovering at this SNR. The total hovering time is calculated using the status data from the AR drone.

\section{Controller with Prior Knowledge}

We used data from 12 flights to evaluate the performance of the controller with the prior knowledge about the SNR to distance mapping. Table 5 summarizes the results and the mean and standard deviation of the SNR indicates the controller's ability to reach the target SNR and stay within the target. However, the $\gamma$ value indicates that the quadcopter is moving back and forth even though it has reached the target SNR.

\begin{tabular}{|l|c|c|l|c|c|}
\hline $\begin{array}{l}\text { Target } \\
\text { SNR } \\
(\mathrm{dB})\end{array}$ & $\begin{array}{c}\text { No.of } \\
\text { Flights }\end{array}$ & $\begin{array}{c}\gamma \\
(\text { Mean })\end{array}$ & $\begin{array}{c}\gamma \\
\text { (Std.Dev) })\end{array}$ & $\begin{array}{c}\text { Achieved } \\
\text { SNR }- \\
\text { Mean } \\
(\mathrm{dB})\end{array}$ & $\begin{array}{c}\text { Achieved } \\
\text { SNR - } \\
\text { Std.Dev }\end{array}$ \\
\hline 30 & 5 & $30.16 \%$ & 0.131 & 30.298 & 0.648 \\
\hline 25 & 7 & $13.63 \%$ & 0.0758 & 25.002 & 0.711 \\
\hline
\end{tabular}

Table 5: Results for the flights with the prior knowledge

The low standard deviation of the SNR indicates that it is fairly stable at the target point. However, we give the target as a single SNR without any tolerance and this causes the controller to move the quadcopter even for minute changes in the SNR. This a reason for the low $\gamma$ and we present an improvement later in this section.

Another possible reason for the low $\gamma$ is the effect of the wind on the quadcopter. The AR Drone that we used is a light-weight quadcopter and we have observed that even slight winds can affect its movements considerably. 


\section{Controller without Prior Knowledge (with the learning module)}

To evaluate the controller's ability to reach the target SNR, and stay at that point, without the prior knowledge we used data from 10 flights. Table 6 summarizes the results of this experiment and they indicate that the controller is capable of reaching the target SNR and stay within that target even without the prior knowledge. The controller learns the SNR to distance mapping while flying the learning algorithm can effectively replace the prior knowledge in guiding the quadcopter towards the target SNR.

\begin{tabular}{|c|c|c|}
\hline Target SNR (dB) & $\begin{array}{c}\text { Achieved SNR - } \\
\text { Mean (dB) }\end{array}$ & $\begin{array}{c}\text { Achieved } \\
\text { SNR- Std. } \\
\text { Dev }\end{array}$ \\
\hline 30 & 29.55716 & 0.585076 \\
\hline 25 & 24.92677 & 0.358824 \\
\hline
\end{tabular}

Table 6: Performance without the prior knowledge

We performed additional experiments to verify whether prior knowledge impacts the time it takes the quadcopter to reach the target SNR. Table 3 shows that with the prior knowledge the quadcopter reaches the target SNR faster. However, note that a flight without the prior knowledge needs a step size which also affects the convergence time.

\section{Improving the stability}

The quadcopter consumes more energy for moving than hovering since more motor thrust is required for movements. Therefore, more hovering time results in less energy consumption. We can reduce the movements due to SNR fluctuations and increase the hovering time by giving the target with a SNR Tolerance $(\beta)$. We used the following algorithm for this purpose.

$\overline{\text { Algorithm } 3 \text { Target with the SNR Tolerance }(\beta)}$
if $($ Target $S N R-\beta)<=$ Current $S N R<=($ Target $S N R+\beta)$ :
HOVER

We gathered data from 5 flights for each value of $\beta,(0,1,2)$, with the target SNR of $25 \mathrm{~dB}$. Table 7 summarizes the results.

\begin{tabular}{|c|c|c|}
\hline$\beta$ & $\gamma($ Mean $)$ & $\gamma$ (Std.Dev) \\
\hline 0 & $13.63 \%$ & 0.0758 \\
\hline 1 & $19.77 \%$ & 0.0622 \\
\hline 2 & $31.37 \%$ & 0.1608 \\
\hline
\end{tabular}

Table 7: Hover measure with different SNR Tolerances

The $\gamma$ increases with the $\beta$. However, with a large $\beta$ the quadcopter may not alway stay at the maximum possible distance from the base station.

\section{CONCLUSIONS AND FUTURE WORK}

We address the problem of hovering a quadcopter at the maximum possible distance from a base station while maintaining a given SNR on the radio link between itself and the base station. We developed a quadcopter controller for this purpose. We improved an initial design through several experiments and finally demonstrated the capability of the controller to reach the target SNR and hover at the point with that SNR.

We intend to use quadcopters equipped with our controller in a wildlife monitoring application. We also plan to further enhance the controller so that it can maintain the radio link quality of a link between two flying quadcopters. Such a controller is useful for maintaining an on air ad hoc network of quadcopters.

\section{REFERENCES}

[1] AR Drone 2.0, UAV. http://ardrone2.parrot.com/.

[2] Calculate distance, bearing and more between Latitude/Longitude points. http://goo.gl/3vA6IW. Accessed: 2015-1-30.

[3] Nouha Baccour, Anis Koubaa, Luca Mottola, Marco Antonio Zuniga, Habib Youssef, Carlo Alberto Boano, and Mario Alves. Radio link quality estimation in wireless sensor networks: a survey. $A C M$ Transactions on Sensor Networks (TOSN), 8(4):34, 2012.

[4] Hubmann Constantin and ÃD̃nderung Letzte. Cognitive Control - An example of Cognitive Dynamic Systems. http://goo.gl/3fFA5i, 2013. Accessed: 2015-1-30.

[5] Kai Daniel, Sebastian Rohde, Niklas Goddemeier, and Christian Wietfeld. Channel Aware mobility for self organizing wireless sensor swarms based on low altitude platforms. In Wireless Communication Systems (ISWCS), 2010 \%th International Symposium on, pages 145-149. IEEE, 2010.

[6] Cory Dixon and Eric W Frew. Maintaining optimal communication chains in robotic sensor networks using mobility control. Mobile Networks and Applications, 14(3):281-291, 2009.

[7] Niklas Goddemeier, Sebastian Rohde, and Christian Wietfeld. Experimental validation of rss driven uav mobility behaviors in ieee 802.11 s networks. In Globecom Workshops (GC Wkshps), 2012 IEEE, pages 1550-1555. IEEE, 2012.

[8] Khim Yee Kam. High bandwidth communications links between heterogeneous autonomous vehicles using sensor network modeling and extremum control approaches. PhD thesis, Monterey, California. Naval Postgraduate School, 2008.

[9] E Pignaton de Freitas, Tales Heimfarth, Ivayr Farah Netto, Carlos Eduardo Lino, Carlos Eduardo Pereira, Armando Morado Ferreira, Flávio Rech Wagner, and Tony Larsson. Uav relay network to support wsn connectivity. In Ultra Modern Telecommunications and Control Systems and Workshops (ICUMT), 2010 International Congress on, pages 309-314. IEEE, 2010.

[10] Sebastian Rohde, Niklas Goddemeier, Kai Daniel, and Christian Wietfeld. Link quality dependent mobility strategies for distributed aerial sensor networks. In GLOBECOM Workshops (GC Wkshps), 2010 IEEE, pages 1783-1787. IEEE, 2010.

[11] Greg Welch and Gary Bishop. An Introduction to the Kalman Filter. Technical report, Chapel Hill, NC, USA, 1995. 